\title{
Chondroblastic osteosarcoma secondary to fibrosarcoma: A case report and literature review
}

\author{
YI-FEI ZHENG, JUN LIN and HUI-LIN YANG
}

Department of Orthopedic Surgery, The First Affiliated Hospital of Soochow University, Suzhou, Jiangsu 215006, P.R. China

Received October 17, 2014; Accepted July 16, 2015

DOI: $10.3892 / \mathrm{ol} .2015 .3792$

\begin{abstract}
Osteosarcoma, which is most common in non-blood systemic tumors, accounts for $20 \%$ of primary bone malignancies. Primary osteosarcoma usually occurs in young individuals aged 10-20 years, while secondary osteosarcoma is more common in the elderly. It had been reported that secondary osteosarcoma may be associated with osteofibrous dysplasia, bone infarction, chondrosarcoma or osteogenesis imperfecta. However, osteosarcoma secondary to fibrosarcoma has rarely been reported. The current study presents the rare case of a female with chondroblastic osteosarcoma secondary to fibrosarcoma. The patient was relatively young and suffered from more than one type of cancer. The course of the disease lasted for $>20$ years. This case study provides general knowledge of osteosarcoma, and discusses its clinical presentation, diagnosis, treatment and prognosis.
\end{abstract}

Introduction. Osteosarcoma is the most common type of primary bone malignancy with an incidence rate of $4-5 \%$ in the USA (1). It most commonly occurs in the femur (42\%), then the tibia $(19 \%)$, the humerus $(10 \%)$, the skull or jaw $(8 \%)$, the pelvis $(8 \%)$, and the ribs $(1.25 \%)$. Osteosarcomas are classified into various subtypes, according to certain characteristics: Intramedullary, cortical, periosteal and parosteal osteosarcoma, according to tumor location; well differentiated or poorly differentiated according to tumor differentiation; osteoblastic, chondroblastic, fibroblastic, telangiectatic and small cell osteosarcoma, according to cell type; and single or multiple, according to tumor number (2). The global incidence of secondary osteosarcoma is $<1 \%$ and surgery is the treatment of choice for this tumor. Patients often exhibit local pain at metastatic sites and symptoms associated with primary disease. Limb salvage surgery may be performed to relieve symptoms of secondary osteosarcoma and to prevent local recurrence (3). Surgery and chemotherapy are used to treat osteosarcoma patients without

Correspondence to: Professor Hui-Lin Yang, Department of Orthopedic Surgery, The First Affiliated Hospital of Soochow University, 188 Shizi Street, Suzhou, Jiangsu 215006, P.R. China E-mail: yhl_sdfyy@163.com

Key words: osteosarcoma, secondary, fibrosarcoma metastasis. However, additional radiotherapy is not recommended for patients without metastasis due to the risk of radiation-induced necrosis of surrounding structures (4). As $80 \%$ of osteosarcoma patients exhibit metastatic disease at diagnosis, the majority are treated with multi-agent chemotherapy in addition to surgical resection. As expected, patients who present with metastases have a worse prognosis and the 5-year survival rate of these patients is only $20-40 \%$ (3). The aim of the present study was to increase knowledge with regard to osteosarcoma and to discuss the clinical presentation, diagnosis, treatment and prognosis of the disease.

\section{Case report}

A 53-year-old female was admitted to the Department of Orthopedics, The First Affiliated Hospital of Soochow University (Suzhou, Jiangsu, China) with increasing pain in the right knee that had been present for 1 month on February 10, 2014. The patient had undergone a lower right femoral tumor resection and knee joint arthrodesis 20 years previously. The patient then received chemotherapy, which consisted of $4.0 \mathrm{~g}$ methotrexate (MTX) and $2.0 \mathrm{mg}$ vincristine (VCR) three times every 2 weeks. The post-operative pathological results showed a fibrosarcoma with muscle and bone destruction. Additional chemotherapy (4.0 g MTX and $2.0 \mathrm{mg}$ VCR) was performed post-operatively. On January 29, 1996, a local lesion resection was performed due to recurrence. The post-operative pathological results confirmed this diagnosis, and the patient subsequently received post-operative chemotherapy $(4.0 \mathrm{~g}$ MTX and $2.0 \mathrm{mg}$ VCR). The patient also received high-dose radiotherapy, with daily doses of radiation of up to $65 \mathrm{~Gy}$ lasting for 5 weeks. On November 25, 1996, a local resection of the lesion was again performed due to recurrence. To complicate matters, the patient also suffered from more than one type of cancer. The patient also underwent surgery due to thyroid papillary carcinoma in 1991. Physical examination showed that there was tenderness and percussion pain on the right knee, and the skin temperature was slightly higher than normal. The movement of the right knee joint was lost.

Upon the current admission, several diagnostic techniques were used, including a blood routine test, a urine routine test, a stool routine test, analysis of the erythrocyte sedimentation rate, $\mathrm{C}$-reactive protein level and serum procalcitonin level, and an electrocardiogram, chest X-ray, and thyroid and inguinal lymph node ultrasound. The results showed an elevated alkaline 


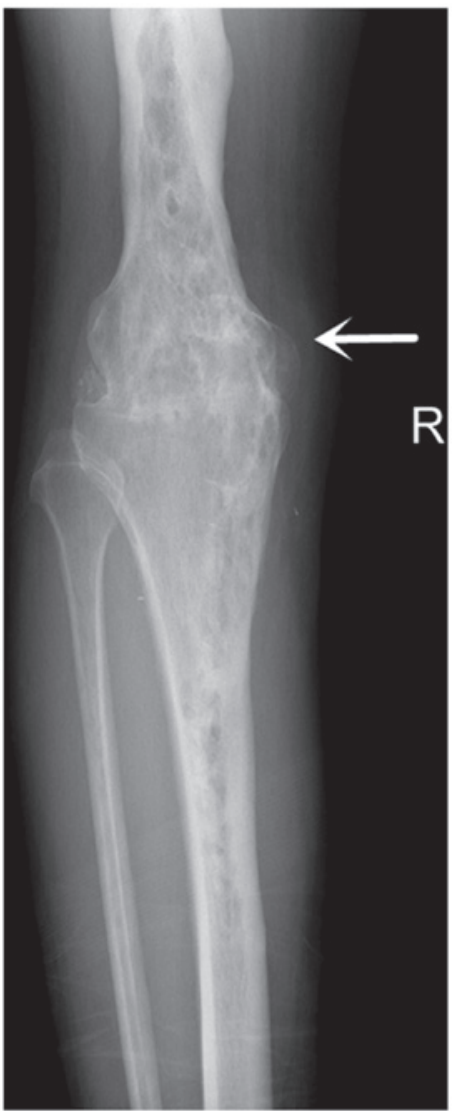

Figure 1. Pre-operative X-ray showing a soft-tissue shadow at the medial side of the distal femur. The arrow indicates the tumor.

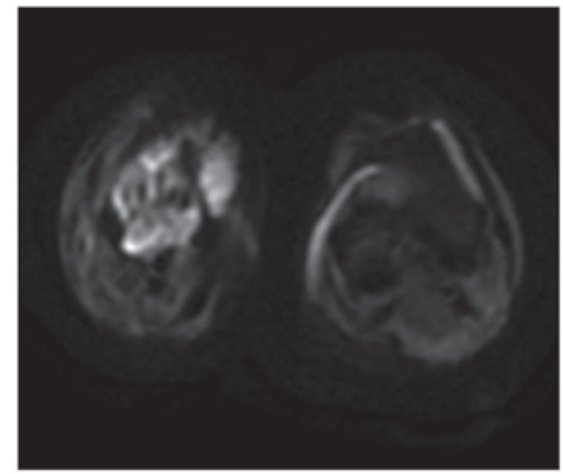

Figure 2. High signal instensity local lesion on diffusion-weighted imaging.

phosphatase level of $192.0 \mathrm{U} / 1$ (normal range, 50-135 U/1). Pre-operative X-ray showed that there was a soft-tissue shadow at the medial side of the distal femur (Fig. 1). Magnetic resonance imaging (MRI) showed the mass with a high signal intensity on diffusion-weighted imaging (Fig. 2), a medium to low signal intensity on T1-weighted imaging (Fig. 3A), high signal intensity on T2-weighted imaging (Fig. 3B), high signal intensity on short time inversion recovery imaging (Fig. 3C), unequal enhancement (Fig. 3D). Furthermore, the results of a computed tomography-guided puncture biopsy were suspicious of chondroblastic osteosarcoma.

On March 5, 2014, the patient underwent a thigh amputation through the right femur. The texture of the resected tumor was relatively soft with a scarlet color. Furthermore, the tumor
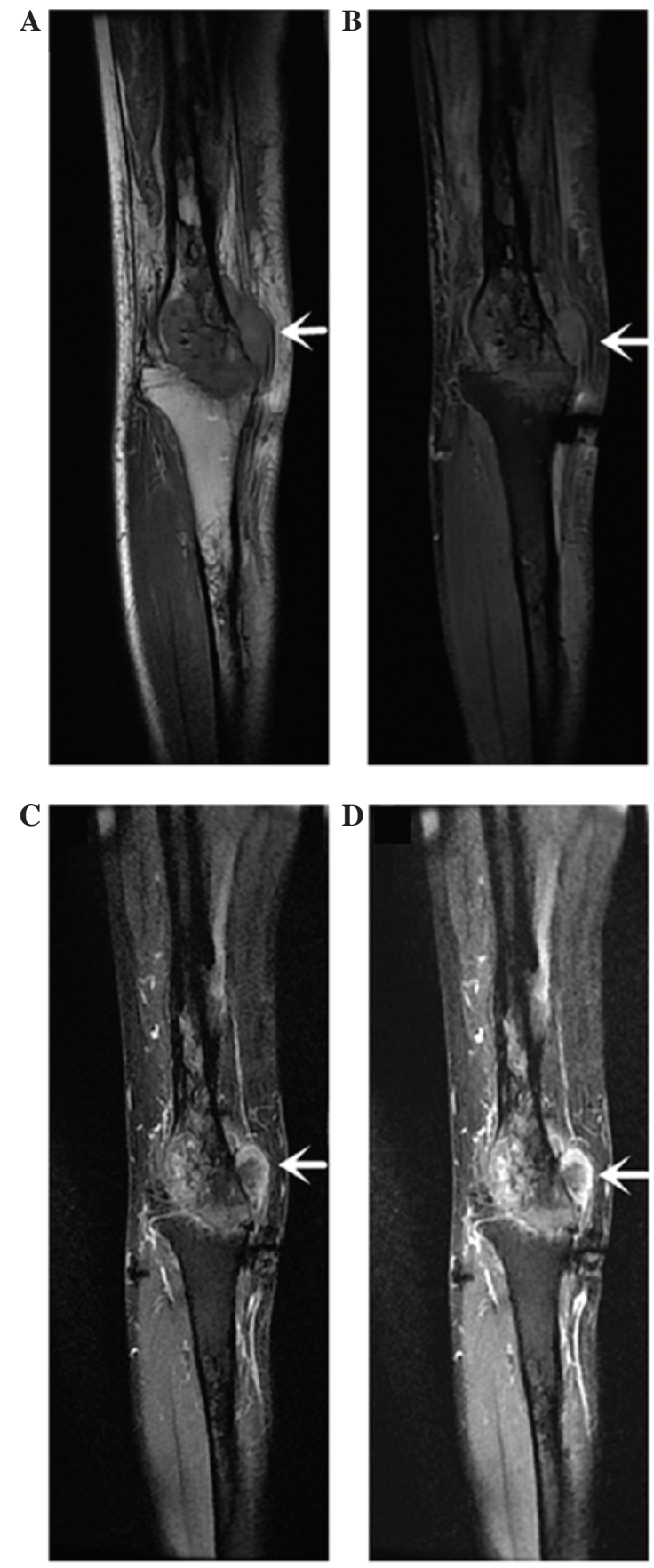

Figure 3. Mass with medium to low signal intsensity on (A) T1-weighted imaging (WI), (B) high signal intensity on T2WI, (C) high signal on short time inversion recovery and (D) unequal enhancement.

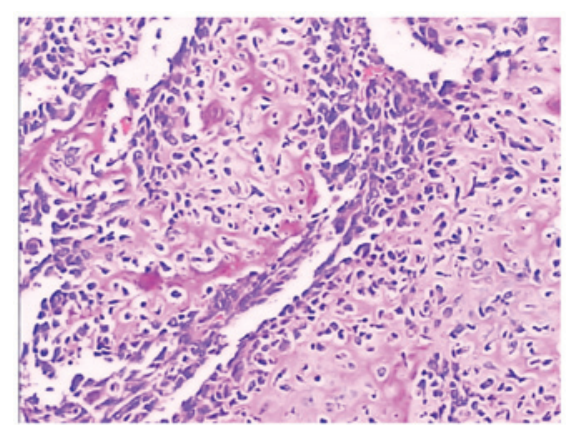

Figure 4. Light micrograph showing a large number of fusiform osteoblastic cells (hematoxylin and eosin staining; original magnification, $\mathrm{x} 20$ ). 
had an irregular shape and measured $1.5 \times 1 \times 3 \mathrm{~cm}^{3}$. The final pathological examination confirmed the diagnosis of a chondroblastic osteosarcoma without involvement of the para-tumor tissues (Fig. 4). Following the surgery, the patient received anti-infection ( $2.0 \mathrm{~g}$ cefonicid sodium) and fluid therapy for 3 days. The patient recovered well, and could walk slowly with a crutch $\sim 3$ weeks after surgery. The patient's condition was discussed with oncologists and subsequently, the patient was administered chemotherapy with methotrexate $(4.0 \mathrm{~g})$ and vincristine $(2.0 \mathrm{mg})$ once, 3 weeks after surgery. In addition, it was recommended that long-term follow-up was continued. Follow-up performed in April 2015 revealed that the patient was well, however the patient subsequently developed lung metastases and refused further treatment. On June 28, 2015, the patient succumbed to the disease due to lung metastasis.

\section{Discussion}

Osteosarcoma, the most common primary malignant tumor that can develop in the bones, can be classified into a number of sub-types, although there is no uniform standard of classification. Previous studies have divided this lesion based on its position, degree of differentiation and components. Osteosarcoma has a well-recognized double peak of incidence, with peak one in children aged 10-14 years, coinciding with the pubertal growth spurt, and peak two in adults aged $>65$ years, which is more likely to be attributed to previous radiotherapy or Paget's disease (4).

In recent years, associated studies have reported that primary osteosarcoma still accounts for the vast majority of primary bone malignancies, while the incidence of secondary osteosarcoma is $<1 \%$. In the present study, a rare case of chondroblastic osteosarcoma secondary to fibrosarcoma is reported. The patient was relatively young and suffered from more than one type of cancer. The course of the disease lasted for $>20$ years.

The aims of modern orthopedic oncology are an optimal tumor resection and a resultant functional residual limb, with increased patient survival (5). With the improvement of technology and treatments, particularly chemotherapy, the opportunity to perform limb salvage has been increased to include $80 \%$ of patients, with an increase in disease-free survival rate from $<20 \%$ prior to the use of effective chemotherapy to $55-75 \%$ following this (6). The advantages and disadvantages of a marginal resection or amputation were therefore carefully analyzed in the present study. In consideration of the gradually improving prostheses and the possibility of local recurrence, an amputation was finally selected. In the view Goorin et al, there is no advantage in event-free survival for patients administered with neoadjuvant chemotherapy (7). However, neoadjuvant chemotherapy can improve the safety of limb salvage (8). The medullary scope of osteosarcoma invasion diagnosed by MRI has clear advantages and has become the main method for determining a reasonable resection plane of bone (9).

Through a review of the pertinent literature, stage can be observed to be the most significant predictive factor, followed by tumor volume, old age, gender and possibly p-glycoprotein expression (10-16). In addition, The present study the response to chemotherapy is the only proven independent factor for the prediction of survival (17). Therefore, after the surgery in the present case, individual chemotherapy and multimodality therapy was performed, as this has been shown to increase the survival rate when compared with surgery alone (18). It is noteworthy that the best time for resuming chemotherapy is within 21 days of definitive surgery. All doctors and patients are required to work together to ensure that chemotherapy is resumed in a timely manner following surgery (19). Osteosarcoma is not sensitive to radiotherapy. For an intralesional surgical margin or where excision is not possible, additional radiotherapy has a positive effect on the prognosis (20). The study by Nakase et al suggested that $\mathrm{p}-53$ gene therapy via cationic liposome modification with transferrin is an effective strategy for the treatment of osteosarcoma. However, further research is required to improve the clinical efficacy (21). Immunotherapy has great potential for treating osteosarcomas, promising improved patient survival rates and quality of life (22).

The current study presents a rare case of osteosarcoma secondary to fibrosarcoma. According to the previous literature, and clinical and radiological findings, this patient could be diagnosed with chondroblastic osteosarcoma. In contrast to other studies, this case is quite rare due to the age of the patient, the fact that the patient suffered from more than one type of cancer and the disease course of $>20$ years. The current study indicates that when a patient with a history of tumor develops clinical symptoms, such as local pain, clinicians should pay attention as this may be an indicator of recurrence or metastasis. Furthermore, the present study indicates that a marginal resection may be a good choice for treating fibrosarcoma. However, when the histopathological result is confirmed as osteosarcoma, amputation may be the better choice of treatment.

\section{Acknowledgements}

This study was supported by the Jiangsu Provincial Special Program of Medical Science (grant no. BL2012004), the Jiangsu Provincial Clinical Orthopedic Center and the Priority Academic Program Development of Jiangsu Higher Education Institutions.

\section{References}

1. Damron TA, Ward WG and Stewart A: Osteosarcoma, chondrosarcoma, and Ewing's sarcoma: National Cancer Data Base Report. Clin Orthop Relat Res 459: 40-47, 2007.

2. Canale ST and Beaty JH (eds): Campbell's Operative Orthopaedics. 12th edition. Elsevier, Philadelphia, PA, 2013.

3. Briccoli A, Rocca M, Salone M, Guzzardella GA, Balladelli A and Bacci G: High grade osteosarcoma of the extremities metastatic to the lung: long-term results in 323 patients treated combining surgery and chemotherapy, 1985-2005. Surg Oncol 19: 193-199, 2010

4. Ottaviani G and Jaffe N: The epidemiology of osteosarcoma. Cancer Treat Res 152: 3-13, 2009.

5. Marulanda GA, Henderson ER, Johnson DA, Letson GD and Cheong D: Orthopedic surgery options for the treatment of primary osteosarcoma. Cancer Control 15: 13-20, 2008.

6. Jaffe N: Osteosarcoma: Review of the past, impact on the future. The American experience. Cancer Treat Res 152: 239-262, 2009.

7. Goorin AM, Schwartzentruber DJ, Devidas M, et al: Presurgical chemotherapy compared with immediate surgery and adjuvant chemotherapy for nonmetastatic osteosarcoma: Pediatric oncology group study POG-8651. J Clin Oncol 21: 1574-1580, 2003.

8. Arpaci F, Ataergin S, Ozet A, et al: The feasibility of neoadjuvant high-dose chemotherapy and autologous peripheral blood stem cell transplantation in patients with nonmetastatic high grade localized osteosarcoma: Results of a phase II study. Cancer 104: 1058-1065, 2005. 
9. Bacci G, Forni C, Longhi A, Ferrari S, Mercuri M, Bertoni F, Serra M, Briccoli A, Balladelli A and Picci P: Local recurrence and local control of non-metastatic osteosarcoma of the extremities: A 27-year experience in a single institution. J Surg Oncol 96: 118-123, 2007.

10. Bramer JA, Van Linge JH, Grimer RJ and Scholten RJ: Prognostic factors in localized extremity osteosarcoma: A systematic review. Eur J Surg Oncol 35: 1030-1036, 2009.

11. Clark JC, Dass CR and Choong PF: A review of clinical and molecular prognostic factors in osteosarcoma. J Cancer Res Clin Oncol 134: 281-297, 2008.

12. Bacci G,Longhi A, Versari M, Mercuri M, Briccoli A and Picci P: Prognostic factors for osteosarcoma of the extremity treated with neoadjuvant chemotherapy 15 -year experience in 789 patients treated at a single institution. Cancer 106: 1154-1161, 2006.

13. Bielack SS, Kempf-Bielack B, Delling G, Exner GU, Flege S, Helmke K, Kotz R, Salzer-Kuntschik M, Werner M, Winkelmann W, et al: Prognostic factors in high-grade osteosarcoma of the extremities or trunk: An analysis of 1,702 patients treated on neoadjuvant cooperative osteosarcoma study group protocols. J Clin Oncol 20: 776-790, 2002.

14. Harting MT, Lally KP, Andrassy RJ, Vaporciyan AA, Cox CS Jr, Hayes-Jordan A and Blakely ML: Age as a prognostic factor for patients with osteosarcoma: An analysis of 438 patients. J Cancer Res Clin Oncol 136: 561-570, 2010.

15. Shalaby S, Shalaby $\mathrm{H}$ and Bassiony A: Limb salvage for osteosarcoma of the distal tibia with resection arthrodesis, autogenous fibular graft and llizarov external fixator. J Bone Joint Surg Br 88 : 1642-1646, 2006.

16. Smeland S, Müller C, Alvegard TA, Wiklund T, Wiebe T, Björk O, Stenwig AE, Willén H, Holmström T, Follerås G, et al: Scandinavian sarcoma group osteosarcoma study SSG VIII: prognostic factors for out-come and the role of replacemen salvage chemotherapy for poor histological responders. Eur J Cancer 39: 488-494, 2003.
17. Davis AM, Bell RS and Goodwin PJ: Prognostic factors in osteosarcoma: A critical review. J Clin Oncol 12: 423-431, 1994.

18. Manoso MW, Healey JH, Boland PJ, Athanasian EA, Maki RG, Huvos AG and Morris CD: De novo osteogenic sarcoma in patients older than forty: Benefit of multimodality therapy. Clin Orthop Relat Res 438: 110-115, 2005.

19. Imran H, Enders F, Krailo M, Sim F, Okuno S, Hawkins D, Neglia J, Randall RL, Womer R, Mascarenhas L and Arndt CA: Effect of time to resumption of chemotherapy after definitive surgery on prognosis for non-metastatic osteosarcoma. J Bone Joint Surg Am 91: 604-612, 2009.

20. Ozaki T, Flege S, Kevric M, Lindner N, Maas R, Delling G, Schwarz R, von Hochstetter AR, Salzer-Kuntschik M, Berdel WE, et al: Osteosarcoma of the pelvis: Experience of the cooperative osteosarcoma study group. J Clin Oncol 21: 334-341, 2003.

21. Nakase M, Inui M, Okumura K, Kamei T, Nakamura S and Tagawa T: P53 gene therapy of human osteosarcoma using a transferrin-modified cationic liposome. Mol Cancer Ther 4: 625-631, 2005.

22. Mori K, Rédini F, Gouin F, Cherrier B and Heymann D: Osteosarcoma: Current status of immunotherapy and future trends (Review). Oncol Rep 15: 693-700, 2006. 